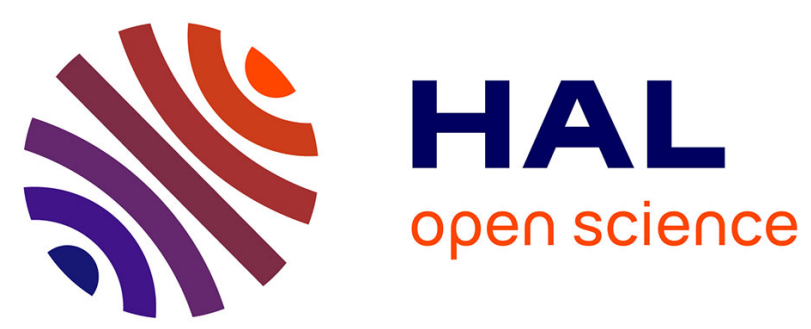

\title{
Bit patterned media based on block copolymer directed assembly with narrow magnetic switching field distribution
}

O Hellwig, J.K. Bosworth, E Dobisz, D Kercher, Thomas Hauet, G Zeltzer, J. D. Risner-Jamtgaard, D Yaney, R Ruiz

\section{To cite this version:}

O Hellwig, J.K. Bosworth, E Dobisz, D Kercher, Thomas Hauet, et al.. Bit patterned media based on block copolymer directed assembly with narrow magnetic switching field distribution. Applied Physics Letters, 2010, 10.1063/1.3293301 . hal-01345344

\author{
HAL Id: hal-01345344 \\ https://hal.science/hal-01345344
}

Submitted on 13 Jul 2016

HAL is a multi-disciplinary open access archive for the deposit and dissemination of scientific research documents, whether they are published or not. The documents may come from teaching and research institutions in France or abroad, or from public or private research centers.
L'archive ouverte pluridisciplinaire HAL, est destinée au dépôt et à la diffusion de documents scientifiques de niveau recherche, publiés ou non, émanant des établissements d'enseignement et de recherche français ou étrangers, des laboratoires publics ou privés. 


\title{
Bit patterned media based on block copolymer directed assembly with narrow magnetic switching field distribution
}

\author{
O. Hellwig, ${ }^{\text {a) }}$ J. K. Bosworth, E. Dobisz, D. Kercher, T. Hauet, G. Zeltzer, \\ J. D. Risner-Jamtgaard, D. Yaney, and R. Ruiz \\ San Jose Research Center, Hitachi Global Storage Technologies, 3403 Yerba Buena Rd., San Jose, \\ California 95135, USA
}

(Received 6 November 2009; accepted 23 December 2009; published online 5 February 2010)

\begin{abstract}
Electron-beam (E-beam) directed assembly, which combines the long-range phase and placement registration of e-beam lithography with the sharp dot size and spacing uniformity of block copolymer self assembly, is considered highly promising for fabricating templates that meet the tight magnetic specifications required for write synchronization in bit patterned media magnetic recording systems. In our study, we show that this approach also yields a narrower magnetic switching field distribution (SFD) than e-beam patterning or block copolymer self-assembly alone. We demonstrate that the pattern uniformity, i.e., island diameter and placement distributions are also important for achieving tight magnetic SFDs. () 2010 American Institute of Physics. [doi:10.1063/1.3293301]
\end{abstract}

Bit patterned media (BPM) magnetic recording systems at densities in excess of $1 \mathrm{~Tb} / \mathrm{in}^{2}$ require an extremely tight lithographic bit placement accuracy and a narrow size distribution of less than $5 \%$ in order to achieve good write synchronization between the recording head and the patterned media. ${ }^{1}$ Nanoimprint technology with master molds fabricated via e-beam directed assembly of block copolymer films is considered a very promising cost-effective approach for creating highly uniform magnetic dot patterns over large areas. ${ }^{2,3}$ In addition to the tight lithographic specifications for BPM, a narrow switching field distribution (SFD) of the magnetic dots is critical to ensure exact bit addressability without overwriting adjacent bits. ${ }^{1,4-7}$

We report magnetic SFD measurements of BPM as a function of pattern uniformity. We use a BPM design based on $\mathrm{Co} / \mathrm{Pd}$ multilayer (ML) magnetic thin films deposited onto nanometer scale pillars formed by substrate etching. ${ }^{4,5,8}$ The nanopillar substrates are fabricated by three different methods: (i) e-beam directed assembly of block copolymer films, ${ }^{3}$ (ii) undirected block copolymer self-assembly, ${ }^{9}$ and (iii) e-beam lithography. ${ }^{5}$ We show that of the three fabrication methods, the combination of e-beam registration and block copolymer self-assembly yields the most uniform pillars, which in turn yield the narrowest magnetic SFD. For less uniform pillars fabricated by e-beam only or by undirected self-assembly alone, we show that nonuniformities in bit size and placement as well as the introduction of defects in the dot lattice (for the case of undirected self assembly) lead to correspondingly broader magnetic SFDs. These results indicate that not only the magnetic media design ${ }^{5}$ and the magnetic reversal process ${ }^{7}$ contribute to the magnetic SFD but the bit size and placement uniformity are also critical for achieving a narrow SFD.

The nanopillar structures were fabricated on $\mathrm{Si}$ substrates using lithographic masks defined by block copolymer films ${ }^{10}$ and by e-beam lithography. The block copolymer templates were made from polystyrene-block-poly(methyl methacrylate) (PS- $b$-PMMA) thin films forming cylindrical PMMA domains oriented perpendicular to the substrate in a

${ }^{a)}$ Electronic mail: olav.hellwig@ @itachigst.com.
PS matrix [Fig. 1(a)]. Polymers with two different molecular weights (both with $\sim 70 \%$ PS content) were used to generate patterns at two different densities. PS-b-PMMA (Polymer Source) with total $M_{n}$ of 65 and $39 \mathrm{~kg} / \mathrm{mol}$ were used to create patterns with a center-to-center pitch of $\mathrm{L}_{\mathrm{o}}=38 \mathrm{~nm}$ $\left(0.5 \mathrm{~Tb} / \mathrm{in}^{2}\right)$ and $\mathrm{L}_{\mathrm{o}}=28 \mathrm{~nm}\left(1.0 \mathrm{~Tb} / \mathrm{in}^{2}\right)$, respectively. $\mathrm{Di}-$ rected assembly of highly uniform patterns with both values of $\mathrm{L}_{\mathrm{o}}$ was achieved by treating the substrates with a polysty-

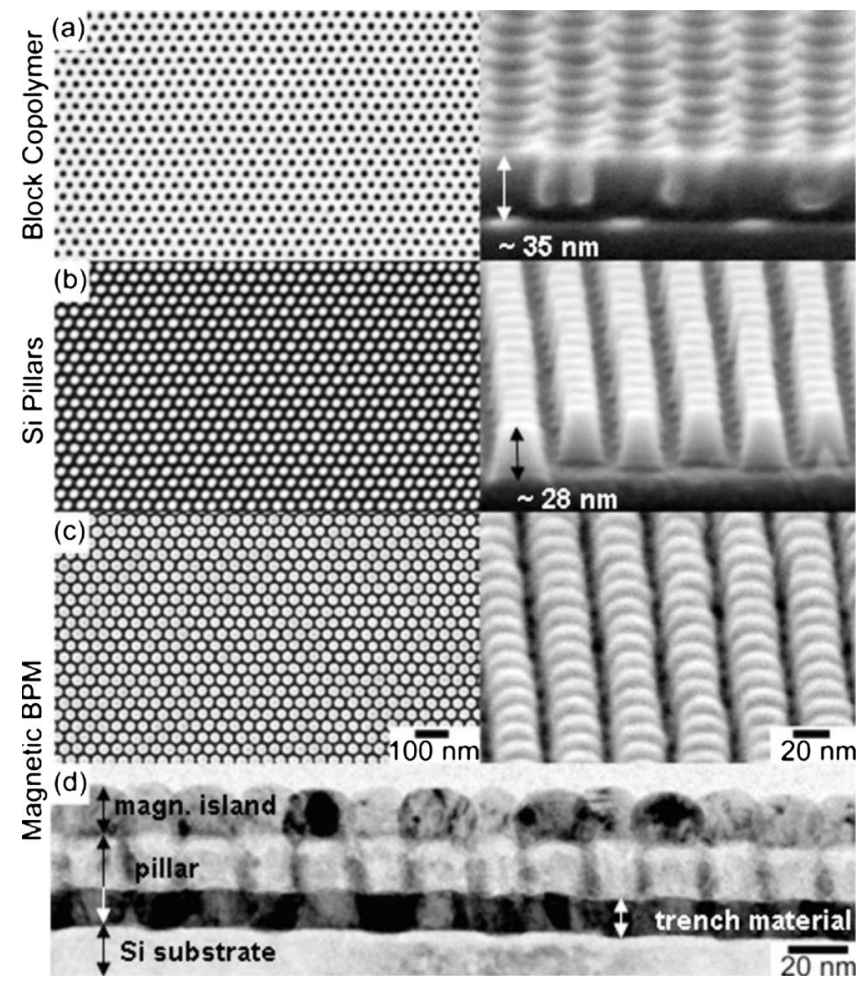

FIG. 1. $0.5 \mathrm{Tbit} / \mathrm{in}^{2}\left(\mathrm{~L}_{\mathrm{o}}=38 \mathrm{~nm}\right) \mathrm{BPM}$ array consisting of MLs deposited onto $\mathrm{Si}$ pillar substrates fabricated via e-beam directed assembly of block copolymer films. SEM micrographs of (a) the block copolymer film after selective removal of the PMMA cylinder cores, (b) Si pillars after Cr lift-off using the template in (a) and subsequent reactive ion etching, and (c) magnetic BPM after depositing a Co/Pd ML thin film onto the pillar structures (left: top view, right: section view at $85^{\circ}$ angle). (d) Bright field TEM crosssectional image through two consecutive rows of bits (into the image plane) that are $180^{\circ}$ phase shifted with respect to each other. 
rene brush and then using e-beam lithography to generate a sparse chemical contrast pattern (density multiplication factor of four). ${ }^{3}$ Undirected, randomly oriented patterns with $\mathrm{L}_{\mathrm{o}}=38 \mathrm{~nm}$ were obtained from block copolymer films applied to substrates with neutral random PS-r-PMMA copolymer brushes, ${ }^{11}$ which induces perpendicular alignment of the PMMA cylinders, but does not otherwise dictate the orientation of the hexagonally packed polymer lattice within the film. Instead the size of the domains and hence defect density and size distribution of PS-b-PMMA perpendicular cylinders were controlled by annealing at different temperatures. Samples annealed at higher temperature displayed a higher degree of ordering with a longer correlation length and a narrower feature size distribution. ${ }^{12,13}$ Finally, we fabricated for comparison pillar templates by e-beam lithography alone on a square lattice with a center-to-center pitch of $35 \mathrm{~nm}$ $\left(530 \mathrm{~Gb} / \mathrm{in}^{2}\right) .^{5}$

Figure 1(a) shows the e-beam directed block copolymer template after selective removal of the PMMA cylinder cores $\left(0.5 \mathrm{~Tb} / \mathrm{in}^{2}\right.$, average diameter $\mathrm{d}=22.9 \mathrm{~nm}$ and normalized standard deviation $\sigma_{\mathrm{d}}=2.6 \%$ ). A Cr lift-off followed by $\mathrm{CF}_{4}$ reactive ion etching ${ }^{3}$ is used to generate Si pillars $20-30 \mathrm{~nm}$ tall with nearly vertical walls and flat tops as shown in Fig. 1(b) $\left(\mathrm{d}=24.1 \mathrm{~nm}\right.$ and $\left.\sigma_{\mathrm{d}}=3.3 \%\right)$. A Ta(15 $\left.\AA\right) / \mathrm{Pd}(30 \AA) /$ $[\mathrm{Co}(2.8 \AA) / \operatorname{Pd}(9 \AA)]_{8} / \operatorname{Pd}(11 \AA)$ ML film is sputter deposited onto the Si pillar substrates. This is done at room temperature and 3 mTorr Ar pressure using deposition rates of $0.7-2 \AA / \mathrm{sec} .^{5,14}$ The magnetic material is deposited on top of the pillars as well as into the trenches. ${ }^{15}$ Corresponding scanning electron microscopy (SEM) and transmission electron microscopy (TEM) images are shown in Figs. 1(c) and $1(\mathrm{~d})$, respectively $\left(\mathrm{d}=29.2 \mathrm{~nm}\right.$ and $\left.\sigma_{\mathrm{d}}=2.8 \%\right)$. The magnetic islands have a larger filling factor with a more spherical overgrown shape resulting from the film growth conditions as seen in cross-sectional SEM [Fig. 1(c)] and TEM images [Fig. 1(d)]. The TEM image also reveals a nearly vertical Si pillar profile with a flat top as well as the presence of trench material. ${ }^{15}$ The magnetic islands are well-decoupled from each other as well as from the trench material as desired for BPM applications. ${ }^{5}$

Figure 2 shows microspot (20-50 $\mu \mathrm{m}$ diameter) polar magneto-optic Kerr effect (PMOKE) hysteresis loops [(a) and (c)] and corresponding island reversal curves [(b) and (d)] for a $0.5 \mathrm{~Tb} / \mathrm{in}^{2}$ [(a) and (b), $\mathrm{L}_{\mathrm{o}}=38 \mathrm{~nm}, \mathrm{~d}=29.2 \mathrm{~nm}$, and $\left.\sigma_{\mathrm{d}}=2.8 \%\right]$ and $1.0 \mathrm{~Tb} / \mathrm{in}^{2}[(\mathrm{c})$ and (d), $28 \mathrm{~nm}$ pitch, d $=20.3 \mathrm{~nm}$, and $\sigma_{\mathrm{d}}=3.2 \%$ ] array aligned to a chemical prepattern. We observe a large reversal step at low fields due to nucleation followed by rapid domain wall motion in the trenches and a relatively small reversal step at high field due to the individual Stoner-Wohlfarth like reversal of the magnetic islands. ${ }^{4,16}$ The relative amplitude of the two reversal steps is not only affected by the relative island/trench filling factor and amount of magnetic material as observed in Figs. 1(c) and 1(d), but also by the optical properties of the trenches and the islands. Also, surface curvature effects, as observed in the TEM image of Fig. 1(d) on top of the pillars (rounded profile), further reduce the reflected signal from the islands. Interference effects between island and trench material typically lead to additional changes in trench and island reversal amplitudes and can even produce a change in sign between island and trench reversal amplitude as observed for the hysteresis loops in Figs. 2(a) and 2(c). ${ }^{4,16}$ However since

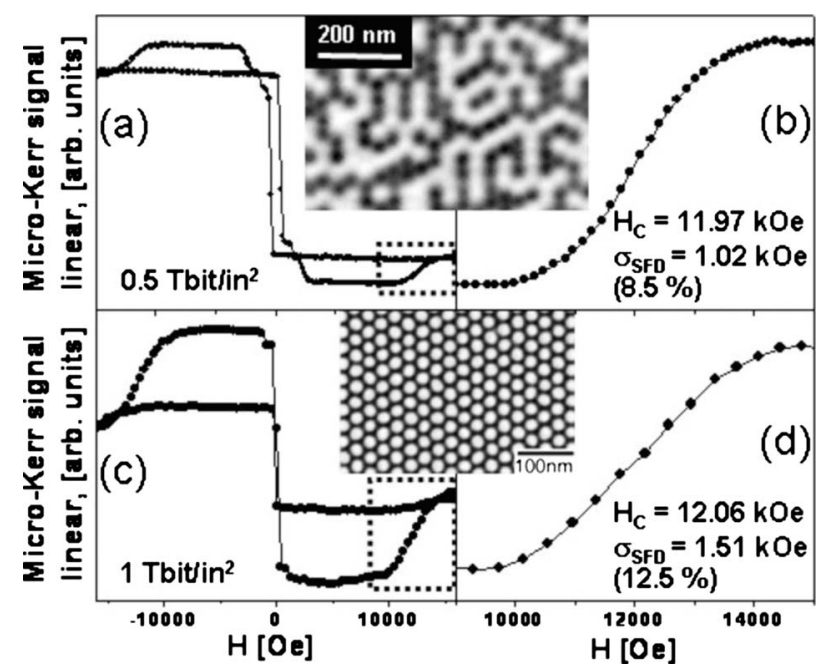

FIG. 2. Microspot PMOKE hysteresis loops [(a) and (c)] and corresponding island reversal curves $[(b)$ and (d)] [enlarged view of the dashed boxes in (a) and (c)] from BPM arrays fabricated via e-beam directed assembly of block copolymer films. (a) and (b) show results from a $0.5 \mathrm{~Tb} / \mathrm{in}^{2}(38 \mathrm{~nm}$ pitch) array, while (c) and (d) were obtained from a $1 \mathrm{~Tb} / \mathrm{in}^{2}(28 \mathrm{~nm}$ pitch) pattern. The insets display a high resolution MFM image of the $0.5 \mathrm{~Tb} / \mathrm{in}^{2}$ array after demagnetization (top) and a SEM image of the $1 \mathrm{~Tb} / \mathrm{in}^{2}$ array after media deposition (bottom), respectively.

island and trench reversal occur in such well-separated field regions we use the island reversal curves in Figs. 2(b) and $2(d)$ to extract the total magnetic SFD $\left(\sigma_{\mathrm{SFD}}\right)$ of the island array, which we define as the standard deviation $\sigma$ of a Gaussian curve fitted to the derivative of the island reversal curve. ${ }^{4,5}$ Corresponding values for the coercivity $\left(\mathrm{H}_{\mathrm{C}}\right), \mathrm{SFD}$ $\left(\sigma_{\mathrm{SFD}}\right)$, and normalized SFD $\left(\sigma_{\mathrm{SFD}} / \mathrm{H}_{\mathrm{C}}\right)$ are displayed in Fig. 2. For the 0.5 and $1 \mathrm{~Tb} / \mathrm{in}^{2}$ arrays we obtain normalized SFDs of $8.5 \%$ and $12.5 \%$, respectively. The inset in Figs. 2(a) and 2(b) shows a high resolution magnetic force microscopy (MFM) image of the $0.5 \mathrm{~Tb} / \mathrm{in}^{2}$ sample after out-ofplane demagnetization. We observe the expected intricate domain structure (each domain is one pillar wide) characteristic of a hexagonal pattern with dipolar interactions between the islands. The inset in Fig. 2(c) is a SEM micrograph of the array at $1 \mathrm{~Tb} / \mathrm{in}^{2}$ after media deposition.

In Fig. 3, we compare the normalized SFD values to BPM sister samples that have been deposited on Si pillar substrates fabricated by e-beam alone. In contrast to the hexagonal e-beam guided block copolymer patterns, we used a

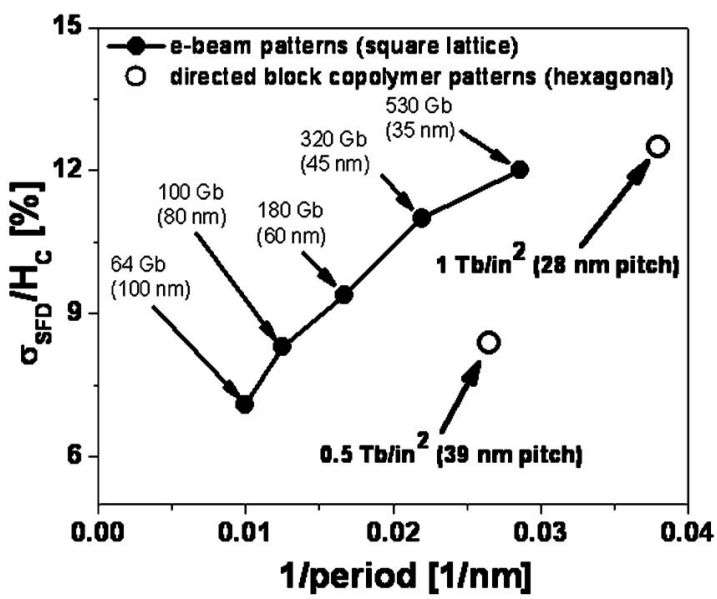

FIG. 3. Normalized SFD vs density for BPM fabricated by e-beam alone (solid circles) and by directed assembly (open circles). 


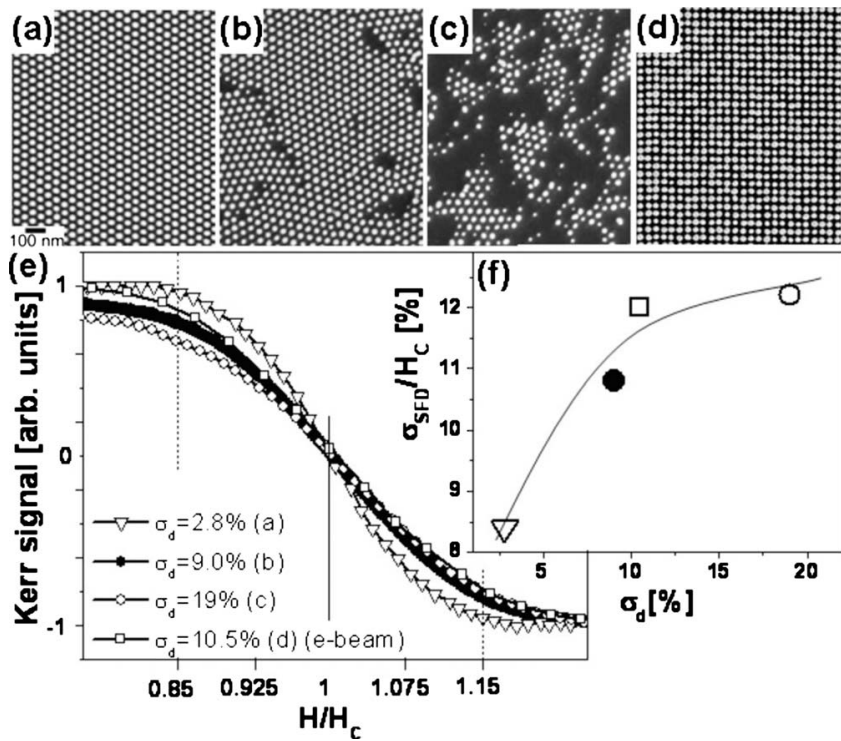

FIG. 4. SEM images of BPM at $0.5 \mathrm{~Tb} / \mathrm{in}^{2}$ with various size distributions fabricated by (a) directed block copolymer assembly, $\sigma_{\mathrm{d}}=2.8 \%$, [(b) and (c)], undirected block copolymer assembly, $\sigma_{\mathrm{d}}=9.0$ and $19 \%$, respectively, and (d) e-beam lithography alone $\sigma_{\mathrm{d}}=10.5 \%$. (e) PMOKE reversal curves of the same four patterns. (f) Normalized magnetic SFD vs pillar size distribution $\sigma_{\mathrm{d}}$ for the four samples. The line is a guide to the eye.

square lattice with a maximum density of $530 \mathrm{~Gb} / \mathrm{in}^{2}$. For conventional e-beam patterns we observe an increase in normalized SFD from about $7 \%$ at $64 \mathrm{~Gb} /$ in $^{2}$ to about $12.2 \%$ at $530 \mathrm{~Gb} / \mathrm{in}^{2}{ }^{5}$. The comparable $0.5 \mathrm{~Tb} / \mathrm{in}^{2}$ pattern from e-beam directed block copolymer assembly reveals a significantly narrower normalized SFD of $8.5 \%$. However, when increasing the density to $1 \mathrm{~Tb} / \mathrm{in}^{2}$, we observe an increase in the SFD, mainly due to the increased dipolar interaction between the dots at the higher areal density ${ }^{14}$ and to some extent also due to the slightly larger size and shape variation as well as defect concentration of the $1 \mathrm{~Tb} / \mathrm{in}^{2}$ pattern.

Finally we compare $0.5 \mathrm{~Tb} / \mathrm{in}^{2}$ magnetic BPM made by directed assembly with media fabricated from undirected block copolymer self-assembly with two different pillar top area size distributions and a pattern fabricated by e-beam lithography alone (Fig. 4). Figure 4(a) shows magnetic islands $\left(\mathrm{d}=29.2 \mathrm{~nm}\right.$ and $\left.\sigma_{\mathrm{d}}=2.8 \%\right)$ obtained from directed assembly of PS-b-PMMA at $\mathrm{L}_{\mathrm{o}}=38 \mathrm{~nm}$. In this sample, all of the hexagonally packed domains of perpendicular cylinders are aligned to form a single coherent lattice. The pillars in Figs. 4(b) and 4(c) were fabricated from undirected PS-bPMMA with $\mathrm{L}_{\mathrm{o}}=38 \mathrm{~nm}$. Two different degrees of ordering and uniformity were obtained by annealing the block copolymer films at two different temperatures. The corresponding block copolymer film used as a template to fabricate the sample in Fig. 4(b) was annealed at $220{ }^{\circ} \mathrm{C}$, yielding larger domain sizes and a narrower size distribution $(\mathrm{d}=25.1 \mathrm{~nm}$ and $\sigma_{\mathrm{d}}=9.0 \%$ ) than the film for the sample in Fig. 4(c), annealed at $190{ }^{\circ} \mathrm{C}\left(\mathrm{d}=21.6 \mathrm{~nm}\right.$ and $\left.\sigma_{\mathrm{d}}=19 \%\right)$. The fabrication of the $\mathrm{Si}$ pillars and subsequent deposition of the $\mathrm{Co} / \mathrm{Pd} \mathrm{ML}$ matched the directed assembly samples as described above. The Cr lift-off process, however, was extremely sensitive to the defect density and domain boundaries resulting in a lower yield of pillar density and a corresponding lower filling factor on the less ordered samples. Figure 4(d) displays a pattern fabricated by e-beam alone for cross comparison.
Figure 4(e) plots the corresponding island reversal curves of the four patterns and Fig. 4(f) summarizes the normalized magnetic SFD as a function of $\sigma_{\mathrm{d}}$. We find that samples made from undirected block copolymer selfassembly or e-beam patterning alone yield a broader magnetic SFD than those made from e-beam directed assembly. Moreover the reversal curves indicate that for the selfassembled structures with multiple domains [Figs. 4(b) and 4(c)] some small fraction of magnetic islands reverse at significantly lower reversal fields than most of the other islands. All reversal curves are normalized with respect to the signal immediately after the trench reversal is completed. We suspect that islands close to the defect lines are responsible for the low field switching tail in the island reversal curves of the self-assembled patterns since they reveal a large variation in diameter, shape, and next nearest neighbor coordination number.

In summary, we report magnetic SFD measurements of BPM deposited on pillar-type substrates with different bit size distributions. We show that the tight size distribution and high uniformity achieved by e-beam directed assembly of block copolymer films can be used to fabricate magnetically narrower SFDs than obtained from e-beam lithography or undirected block copolymer self-assembly alone. Our study shows that not only the magnetic media design ${ }^{5}$ and the magnetic reversal process ${ }^{7}$ contribute to the magnetic SFD, but that also pattern uniformity of island diameter and spacing are important factors. In addition to delivering the tight tolerances in island diameter and island position required for BPM write synchronization, templates made by directed assembly also yield a tighter magnetic SFD, thus providing a promising pathway for BPM fabrication at densities of $1 \mathrm{~Tb} / \mathrm{in}^{2}$ and beyond.

${ }^{1}$ M. E. Schabes, J. Magn. Magn. Mater. 320, 2880 (2008).

${ }^{2}$ B. D. Terris and T. Thomson, J. Phys. D: Appl. Phys. 38, R199 (2005). ${ }^{3}$ R. Ruiz, H. Kang, F. A. Detcheverry, E. Dobisz, D. S. Kercher, T. R. Albrecht, J. J. de Pablo, and P. F. Nealey, Science 321, 936 (2008).

${ }^{4}$ O. Hellwig, A. Berger, T. Thomson, E. Dobisz, H. Yang, Z. Bandic, D. Kercher, and E. E. Fullerton, Appl. Phys. Lett. 90, 162516 (2007).

${ }^{5}$ O. Hellwig, T. Hauet, T. Thomson, E. Dobisz, J. D. Risner-Jamtgaard, D. Yaney, B. D. Terris, and E. E. Fullerton, Appl. Phys. Lett. 95, 232505 (2009).

${ }^{6}$ J. M. Shaw, S. E. Russek, T. Thomson, M. J. Donahue, B. D. Terris, O. Hellwig, E. Dobisz, and M. L. Schneider, Phys. Rev. B 78, 024414 (2008).

${ }^{7}$ T. Hauet, E. Dobisz, S. Florez, J. Park, B. Lengsfield, B.D. Terris and O. Hellwig, Appl. Phys. Lett. 95, 262504 (2009).

${ }^{8}$ T. Thomson, G. Hu, and B. D. Terris, Phys. Rev. Lett. 96, 257204 (2006).

${ }^{9}$ K. W. Guarini, C. T. Black, and S. H. I. Yeuing, Adv. Mater. 14, 1290 (2002).

${ }^{10}$ For patterned media and template fabrication by block copolymers, see C. A. Ross and J. Y. Cheng, MRS Bull. 33, 838 (2008); C. T. Black, R. Ruiz, G. Breyta, J. Y. Cheng, M. C. Colburn, K. W. Guarini, H.-C. Kim, and Y. Zhang, IBM J. Res. Dev. 51, 605 (2007), and references therein.

${ }^{11}$ P. Mansky, Y. Liu, E. Huang, T. P. Russell, and C. J. Hawker, Science 275, 1458 (1997).

${ }^{12}$ R. Ruiz, J. K. Bosworth, and C. T. Black, Phys. Rev. B 77, 054204 (2008).

${ }^{13}$ M. R. Hammond, S. W. Sides, G. H. Fredrickson, E. J. Kramer, J. Ruokolainen, and S. F. Hahn, Macromolecules 36, 8712 (2003).

${ }^{14}$ O. Hellwig, J. B. Kortright, A. Berger, and E. E. Fullerton, J. Magn. Magn. Mater. 319, 13 (2007).

${ }^{15}$ O. Hellwig, A. Moser, E. Dobisz, Z. Bandic, H. Yang, D. S. Kercher, J. D. Risner-Jamtgaard, D. Yaney, and E. E. Fullerton, Appl. Phys. Lett. 93, 192501 (2008).

${ }^{16}$ V. Baltz, B. Rodmacq, A. Bollero, J. Ferré, S. Landis, and B. Dieny, Appl. Phys. Lett. 94, 052503 (2009). 